\title{
Risk Factors: Pathogenesis, Diagnosis and Management of Delirium
}

\author{
Haxhire Gani \\ UHC. "Mother Teresa”, Dept. of Surgery, Tirana, Albania
}

\begin{abstract}
Delirium is a change in mental status characterized by a reduced awareness of the environment and a disturbance in attention. Pathogenesis: The underlying mechanisms contributing to delirium are poorly understood. Risk factors for delirium The following are risk factors which are associated with an increased risk of delirium: Age $\geq 65$ years. Male sex. Pre-existing cognitive deficit, e.g. dementia, stroke. Severity of dementia. Severe comorbidity etc. Medical management: Using drugs to treat delirium can lead to adverse effects and worsening of delirium; therefore, careful consideration is required.
\end{abstract}

Keywords: Delirium, Dementia, Depression Bipolar Disorder

\section{Introduction}

Delirium is well defined and is described in the Diagnostic and Statistical Manual of Mental Disorders fourth edition (DSM-IV-TR; ). The key characteristics are a change in mental status characterized by a reduced awareness of the environment and a disturbance in attention. This may be accompanied by other, more florid, perceptual symptoms (hallucinations) or cognitive symptoms including disorientation or temporary memory dysfunction. The patient may express hypoactive, hyperactive, or mixed psychomotor behaviours. Several tests have been developed and validated for use in diagnosis and grading of delirium. Delirium is a clinical syndrome which is difficult to define exactly but involves abnormalities of thought, perception and levels of awareness. Delirium is a frequent post operative disorder to the sick and elderly, and always has been associated with extent of day stay in hospital, and certainly more cost for the hospital as well. It typically is of acute onset and intermittent. ${ }^{1}$ Both hypoactive and hyperactive delirium states are recognized and often patients exhibit features of both. $\underline{\mathbf{1}}$ Patients may appear confused or "not with it" when talking to them. Alternatively, it may be their family or carer noticing the confusion. Delirium is the most common complication of hospitalisation in the elderly population $\stackrel{2}{2}$.Delirium occurs in about $15-20 \%$ of all

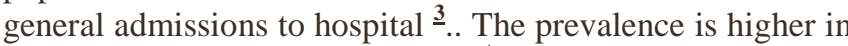
patients with malignancy and $\mathrm{HIV}^{4}$.

Pathogenesis: These models, along with clinical studies of drugs with ,anticholinergic activity ${ }^{\text {ee }}$ have contributed to a hypocholinergic theory of delirium. $\underline{5}$

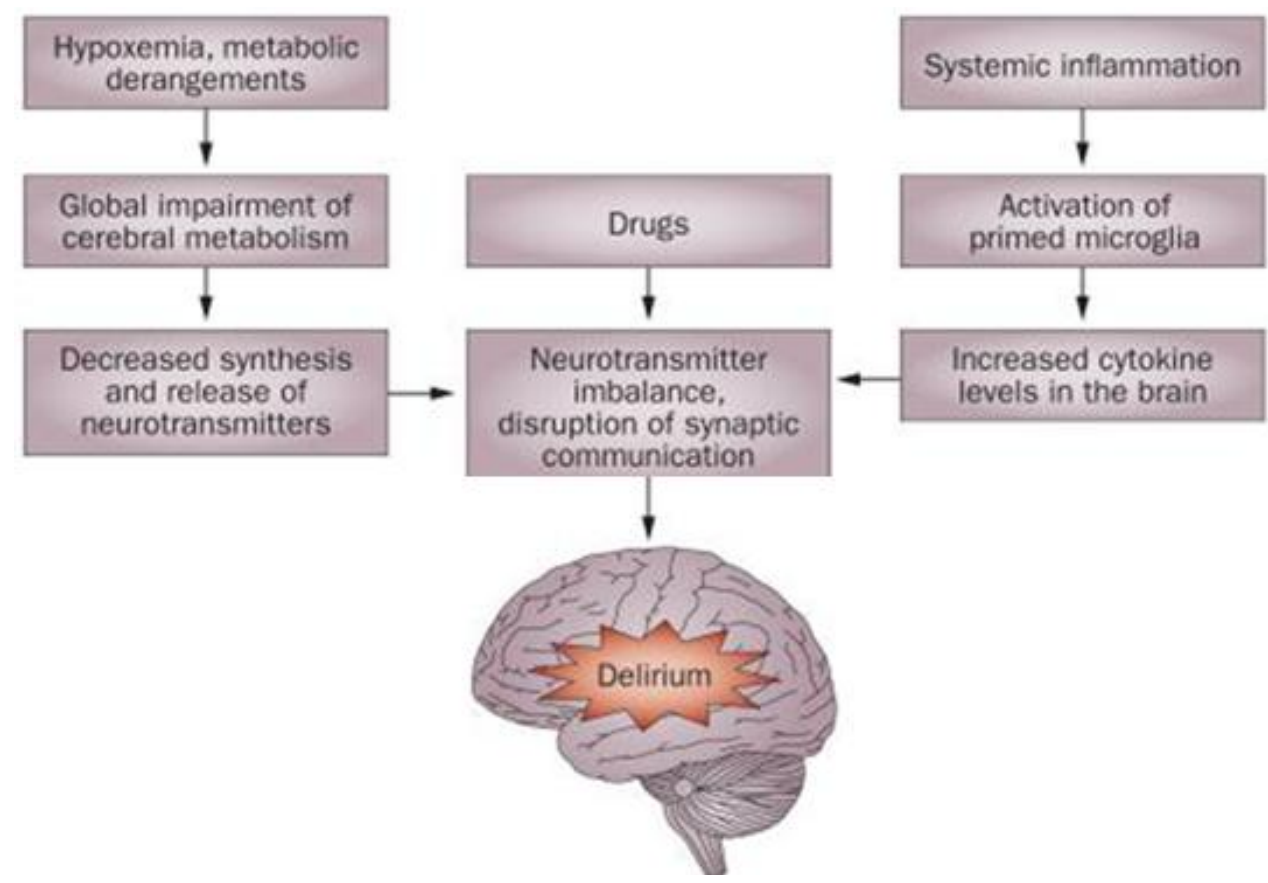

Figure 1: Relationships between various etiological factors in delirium. Systemic inflammation can be the result of systemic infection, trauma or surgery. Neurotransmitters with possible roles in delirium include acetylcholine, dopamine, 5hydroxytryptamine, norepinephrine 


\section{International Journal of Science and Research (IJSR) \\ ISSN (Online): 2319-7064}

Index Copernicus Value (2013): 6.14 | Impact Factor (2015): 6.39

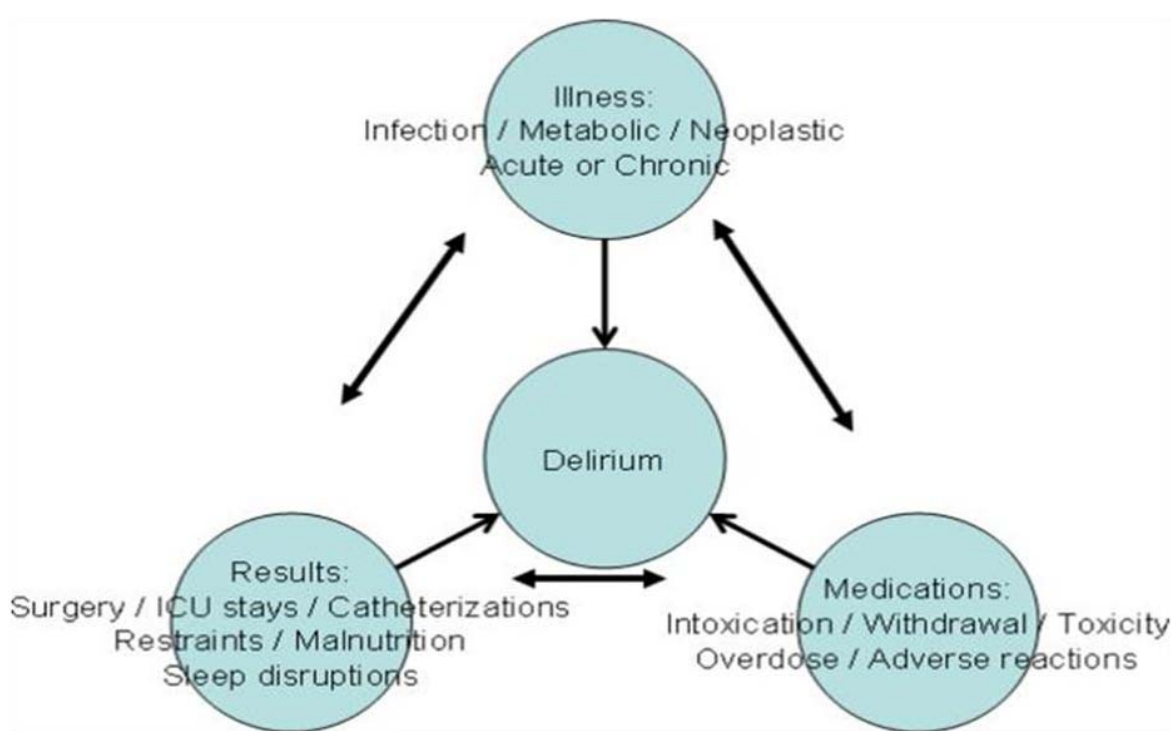

Figure 2: Illness is the prerequisite for delirium, but the medications used to combat illness and the resulting interventions can also lead to delirium, and interact with each other to cause delirium ${ }^{-6}$

The underlying mechanisms contributing to delirium are poorly understood. Many theories emphasize aberrant neurotransmission. One of the most widely accepted mechanisms is cholinergic deficiency; increased serum anticholinergic activity is associated with delirium. ${ }^{7}$ Other hypotheses invoke abnormalities in melatonin and serotonin,,${ }^{8,9}$ with abnormal tryptophan metabolism unifying these ideas because tryptophan is Neuronal damage is an alternative explanation, secondary either to oxidative stress ${ }^{10}$ or inflammation. Proinflammatory cytokines increase in postoperative delirium, 11 especially interleukin-6 and interleukin-8. ${ }^{12}$.In addition, elevations in C-reactive protein occur in delirious patients. A link between inflammation and neurotransmission has been proposed, with inflammationinduced perivascular edema leading to hypoxia and subsequent reduced synthesis of acetylcholine. $\underline{13}$ It is generally thought that delirium represents global brain dysfunction. Electroencephalographic findings reveal a decrease in the fast alpha frequencies and an increase in the slower theta rhythm. $\underline{14}$ In hypoactive delirium, hypoperfusion occurs globally in the frontal, temporal, and occipital lobes, and focally in the caudatehead, thalamus, and lenticular nuclei. Delirium improves once blood flow returns to normal, suggesting that cerebral hypoperfusion may play a role.$\underline{15}$

\section{Risk factors for delirium}

1,3,16,17,18,19 The following are risk factors which are associated with an increased risk of delirium:

Age $\geq 65$ years

Male sex

Pre-existing cognitive deficit, e.g. dementia, stroke. Severity of dementia. Severe comorbidity. Previous episode of delirium. Operative factors, e.g. type of operation - hip fracture repairs are more likely to be associated with delirium, as are emergency operations. Certain conditions burns, AIDS, fractures, infection, low albumin, dehydration. Current hip fracture or severe illness. ${ }^{1}$ Drug use (implicated in nearly half of cases) and dependence, e.g. benzodiazepines. Substance misuse, e.g. alcohol. Extremes of sensory experience, e.g. hypothermia or hyperthermia. Visual or hearing problems. Poor mobility. Social isolation. Stress. Terminally ill. Movement to a new environment.ICU admission. High serum urea levels have also been found to be a risk factor for delirium in acutely admitted patients in one prospective study. ${ }^{20}$ Usually a precipitant is required along with risk factors for delirium to occur. Further, the presence of a greater number of risk factors to begin with means that only a small precipitant is necessary to trigger delirium.

Causes of delirium ${ }^{4,17,19,21}$ Acute infections: Urinary tract infection. Pneumonia. Sepsis. Viral infections. Meningitis. Encephalitis. Cerebral abscess. Malaria.

Prescribed drugs: Benzodiazepines. Analgesics, e.g. morphine. Anticholinergics. Anticonvulsants. AntiParkinsonism medications. Steroids.

\section{Surgical: Postoperative.}

\section{Toxic substances}

Substance misuse or withdrawal. Alcohol - acute intoxication or withdrawal. Carbon monoxide (CO) poisoning. Exposure to heavy metals. Barbiturate withdrawal.

Vascular disorders: Cerebrovascular haemorrhage or infarction. Cardiac failure or is chaemia. Subdural haemorrhage. Subarachnoid haemorrhage. Vasculitis, e.g. systemic lupus erythematosus (SLE).Cerebral venous thrombosis. Migraines.

Metabolic causes: Hypoxia. Electrolyte abnormalities, e.g. hyponatraemia and hypercalcaemia. Hypoglycaemia or hyperglycaemia. Hepatic impairment. Renal impairment.

Vitamin deficiencies: Thiamine deficiency. Nicotinic acid deficiency. Vitamin B12 deficiency. 


\section{International Journal of Science and Research (IJSR) \\ ISSN (Online): 2319-7064 \\ Index Copernicus Value (2013): 6.14 | Impact Factor (2015): 6.39}

Endocrinopathies: Hypothyroidism and hyperthyroidism. Hypopituitarism. Hypoparathyroidism hyperparathyroidism. Cushing's disease. Porphyria. Carcinoid.

Trauma: Head injury.

Epilepsy: For example, postictally.

Neoplasia: Primary cerebral malignancy. Secondaries in the brain. Paraneoplastic syndromes.

Others: Urinary retention. Faecal impaction.

Multiple aetiology.

Unknown aetiology.

The most common causes are medical conditions such as infections, medications or drug withdrawal. The diagnosis of delirium is clinical. The following features may be present: Usually acute or subacute presentation. Fluctuating course. Consciousness is clouded/impaired cognition/disorientation. Poor concentration. Memory deficits - predominantly poor short-term memory. Abnormalities of sleep-wake cycle, including sleeping in the day. Abnormalities of perception, e.g. hallucinations or illusions.Agitation. Emotional lability. Psychotic ideas are common but of short duration and of simple content. Neurological signs, e.g. unsteady gait and tremor. Only some of these symptoms may be present. The symptoms may coincide with underlying dementia - which is common. The diagnosis is still clinical and criteria are set out by the Diagnostic and Statistical Manual of Mental Disorders (DSM-IV) as illustrated in the next section.

DSM diagnosis of delirium due to a medical condition $\underline{22}$ Disturbance of consciousness with decreased clarity of awareness and difficulties of attention.Change in cognition, e.g. memory deficit and disorientation or presence of perceptual abnormalities. These changes are not the result of previous or evolving dementia. The disturbance develops over a short period of time and fluctuates. There is evidence that the disturbance is the result of a general medical condition ,

Subtypes of delirium ${ }^{4}$ Hypoactive subtype - apathy and quiet confusion are present and easily missed. This type can be confused with depression. Hyperactive subtype agitation, delusions and disorientation are prominent and it can be confused with schizophrenia. Mixed subtype patients vary from hypoactive to hyperactive. Assessment. Check: Airway/breathing/circulation. Conscious level. Vital signs, e.g. pulse oximetry, pulse, blood pressure, temperature. Capillary blood glucose. Full cardiovascular and respiratory examination. Full abdominal and genitourinary examination, if appropriate. Full neurological examination. Further examination depending on the suspected problem, e.g. ENT or rectal examination.

Differential diagnosis $\frac{17}{}$. Delirium is commonly mistaken for the following diagnoses: Dementia ,Depression Bipolar Disorder .Functional psychoses, e.g. Schizophrenia .
Investigations: These should be guided by the clinical presentation and are aimed at identifying an underlying cause of the delirium. Typical investigations that can be performed include:

Full history, including collateral history and cognition testing, e.g. mini mental state examination. Full examination - look for sources of infection, including the ears and throat; look for rashes, lymphadenopathy and check for constipation. Bloods - include FBC, U\&Es and creatinine, glucose, calcium, magnesium, LFTs, TFTs, cardiac enzymes, vitamin B12 levels, syphilis serology, autoantibody screen and PSA. Creatinine is vital to obtain an estimated glomerular filtration rate (eGFR), as this may indicate impaired renal function and affect the handling of medications, and may predispose to drug-induced delirium. Urine dipstick testing and microscopy. Blood cultures and serology, if indicated. ECG. Pulse oximetry and arterial blood gas, if indicated.CXR and possibly abdominal X ray, if indicated. Further imaging, e.g. CT scan of the brain. Lumbar puncture may be necessary.EEG - this is usually only performed if there is doubt regarding the diagnosis, and shows generalised diffuse slowing in $80 \%$ of delirious patients.

Management $\frac{3,4,17}{2}$ This begins with increased awareness of delirium and regular measures of cognitive function. The underlying cause needs to be treated. It is common for patients with delirium to be admitted to hospital to help investigate the patient and for supportive management. However, some patients can be managed in the community and moving patients to a new environment can worsen delirium. In delirium, the features are fluctuating and some patients are lucid between episodes and can thus provide informed consent during these periods. However, if the patient is not able to provide informed consent then they can be treated in their best interests under common law. But more specific to delirium, the management can be divided into: Supportive management. Environmental measures. Medical management. Management post-discharge.

Management: Clear communication. Reminders of the day, time, location and identification of surrounding persons. Have a clock available. Have familiar objects from home around patients, especially glasses, walking aids and hearing aids. Staff consistency - both doctors and nurses. Relaxation, e.g. watch television. Involve the family and careers.

\section{Circumstantial measures}

Avoid sensory extremes (over- or understimulation).Adequate space and sleep. Single rooms if possible. Avoid specialty jargon. Control excess noise.

Control room lighting and have a low wattage bulb at night. Control room temperature (aim for $21-23^{\circ} \mathrm{C}$ ). Use health advocates (interpreters) where needed and if possible. Maintain competence, e.g. maintain walking in ambulant patients. Adequate nutrition and attention to continence.

\section{A note on managing wandering}

The delirious patient may have a tendency to wander. It is common to think of restraining and/or sedating the patient in these circumstances. However, this may only worsen the 


\section{International Journal of Science and Research (IJSR) \\ ISSN (Online): 2319-7064}

Index Copernicus Value (2013): 6.14 | Impact Factor (2015): 6.39

situation. The management should aim to keep the patient safe using the least restrictive management, e.g. think of causes of agitation or wandering (e.g. need for toilet). These causes should be rectified; if this is not possible, using distraction may help. Relatives may be helpful in this scenario.

\section{Medical management}

Using drugs to treat delirium can lead to adverse effects and worsening of delirium; therefore, careful consideration is required. Antipsychotics $\stackrel{2}{=}$ have beneficial effects in selected patients who are agitated and their onset is relatively fast. Haloperidol_is preferred, although there is a risk of extrapyramidal side-effects (EPSEs). Small doses should be used, e.g. 0.5-2.0 mg PO/IM/IV. It may be appropriate to consider a lower dose in elderly patients, e.g. $0.5 \mathrm{mg}$. This can be repeated after 30 minutes if there is no response. Lorazepam is generally the first choice of benzodiazepines, as it has a rapid onset of action and a short duration. Lorazepam can be used alone or with low doses of haloperidol if there are concerns regarding EPSEs, e.g. Parkinson's disease or Lewy body dementia. A starting dose of $0.5-1 \mathrm{mg}$ PO can be given 2-hourly or $0.5-1 \mathrm{mg}$ IV/IM (both to a maximum of $3 \mathrm{mg}$ in a 24-hour period). Atypical antipsychotics have also been used; however, olanzapine and risperidone should be avoided in the elderly, as they have been associated with an increased risk of cerebrovascular disease $\stackrel{2}{ }$.

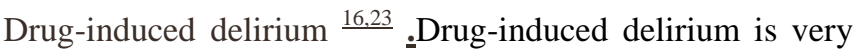
common amongst the elderly. Drugs can be the sole cause of delirium in some. Common drug causes of delirium include: Benzodiazepines.Narcotic analgesics, antihistamines. Antispasmodics. Flouroquinolones. Warfarin. Captopril. Theophylline. Isosorbide dinitrate. Dipyridamole. Furosemide. Lithium. Tricyclic antidepressants. Cimetidine. Anti-arrhythmics. Statins. $\stackrel{24}{\text {.Digoxin, Steroids. }}$ Betablockers.Over-the-counter medications, e.g. liquid medications containing alcohol or chlorphenamine. The role of medications may be suggested by a temporal relationship between onset of delirium and start of new medication. However, this is not always the case and practitioners need to be aware of this. Medication lists should be thoroughly reviewed in delirium. The exact mechanism of delirium is unclear but it is postulated that central cholinergic pathway blockade is a major factor. ${ }^{23}$ This may explain why anticholinergic medications readily lead to delirious states. It may be that this factor along with the pharmacokinetic changes that occur later in life and comorbidities increase the susceptibility of elderly patients to drug-induced delirium. Management involves stopping the offending drug; however, the actual causal medication is often unknown. In this case, all unnecessary medications should be stopped or doses reduced. These medications can be increased or reintroduced when the patient has improved. Furthermore, it may be prudent to prescribe alternatives to medications with high anticholinergic activity, e.g. use of proton pump inhibitors rather than cimetidine. $\underline{\underline{23}}^{2}$

\section{References}

[1] Delirium ,NICE Clinical Guideline (July 2010); Delirium: diagnosis, prevention and management

[2] Young J,Inouye SK: Delirium in older people. BMJ 2007;334:842-846

[3] Meagher DJ: Delirium: optimising management. BMJ. 2001 Jan 20;322(7279):144-9.

[4] Gleason OC:Delirium. Am Fam Physician. 2003 Mar 1;67(5):1027-34. [abstract]

[5] Hshieh, TT; Fong, TG; Marcantonio, ER; Inouye, SK (July 2008). "Cholinergic deficiency hypothesis in delirium: a synthesis of current evidence.". The journals of gerontology. Series A, Biological sciences and medical sciences 63 (7): 764-72. doi:10.1093/gerona/63.7.764.PMC: 2917793.PMID 18693233.

[6] Dennis M. Popeo,Delirium in older adults,.. Mt Sinai J Med. ;2011 Jul-Aug;78(4):571-82. doi: 10.1002/msj.20267,78(4):571-582.

[7] Mussi C, Ferrari R, Ascari S, et al. Importance of serum anticholinergic activity in the assessment of elderly patients with delirium. J Geriatr Psychiatry Neurol 1999;12(2):82-6.

[8] Uchida K, Aoki T, Ishizuka B. Postoperative delirium and plas Hypotheses 1999;53(2):103-6.

[9] Balan S, Leibovitz A, Zila SO, et al. The relation between the clinical subtypes of delirium and the urinary level of 6-SMT. J Neuropsychiatry Clin Neurosci 2003; 15(3):363-6.

[10] Karlidag R, Unal S, Sezer $\mathrm{OH}$, et al. The role of oxidative stress in postoperative delirium. Gen Hosp Psychiatry 2006;28(5):418-23.

[11] Rudolph JL, Ramlawi B, Kuchel GA, et al. Chemokines are associated with delirium after cardiac surgery. J Gerontol A Biol Sci Med Sci 2008;63A(2):184-9

[12] de Rooij SE, van Munster BC, Korevaar JC, et al. Cytokines and acute phase response in delirium. J Psychosom Res 2007;62(5):521-5.

[13] Hala M. Pathophysiology of postoperative delirium: systemic inflammation as a response to surgical trauma causes diffuse microcirculatory impairment. Med Hypotheses 2007;68(1):194-6.

[14] Plaschke K, Hill H, Engelhardt R, et al. EEG changes and serum anticholinergic activity measured in patients with delirium in the intensive care unit. Anaesthesia 2007;62(12):1217-23.

[15] Yokota H, Ogawa S, Kurokawa A, et al. Regional cerebral blood flow in delirium patients. Psychiatry Clin Neurosci 2003;57(3):337-9.

[16]Burns A,Gallagley A,Byrney J: Delirium. J Neurol Neurosurg Psychiatry. 2004 Mar;75(3):362-7. [abstract]

[17] Brown TM,Boyle MF;Delirium. BMJ. 2002 Sep 21;325(7365):644-7.

[18] Agnoletti V,Ansaloni L,Catena,et al; Postoperative Delirium after elective and emergency surgery: analysis and checking of risk factors. A study protocol. BMC Surg. 2005 May 28;5:12. [abstract]

[19] Kumar, P. and Clark, M. (2005) Clinical Medicine, 6th Edition, Elsevier Limited

[20] Korevaar JC,van Munster BC,de Rooij SE;Risk factors for delirium in acutely admitted elderly patients: a 


\section{International Journal of Science and Research (IJSR) \\ ISSN (Online): 2319-7064}

Index Copernicus Value (2013): 6.14 | Impact Factor (2015): 6.39

prospective cohort study. BMC Geriatr. 2005 Apr 13;5:6. [abstract]

[21] Boon, N.A., Colledge, N.R. and Walker, B.R. (2006)

Davidscon's Principles and Practice of Medicine 20th Edition, Elsevier Limited

[22] Delirium; Diagnostic and Statistical Manual of Mental Disorders

[23] Alagjakrishnan K,Wiens CA; An approach to drug induced delirium in the elderly. Postgrad Med J. 2004 Jul;80(945):388-93. [abstract]

[24] Redelmeier DA,Thiruchelvam D,Daneman N;Delirium after elective surgery among elderly patients taking statins. CMAJ. 2008 Sep 23;179(7):645-52. [abstract] 